\title{
A novel de novo mutation in MYT1, the unique OAVS gene identified so far
}

\author{
Marie Berenguer ${ }^{1}$, Angele Tingaud-Sequeira ${ }^{1}$, Mileny Colovati ${ }^{2}$, Maria I Melaragno ${ }^{2}$, Silvia Bragagnolo ${ }^{2}$, \\ Ana BA Perez ${ }^{2}$, Benoit Arveiler ${ }^{1,3}$, Didier Lacombe ${ }^{1,3}$ and Caroline Rooryck ${ }^{\star, 1,3}$
}

Oculo-auriculo-vertebral spectrum (OAVS) is a developmental disorder characterized by hemifacial microsomia associated with ear, eyes and vertebrae malformations showing highly variable expressivity. Recently, MYT1, encoding the myelin transcription factor 1, was reported as the first gene involved in OAVS, within the retinoic acid (RA) pathway. Fifty-seven OAVS patients originating from Brazil were screened for MYT1 variants. A novel de novo missense variant affecting function, $c .323 \mathrm{C}>\mathrm{T}$ ( $\mathrm{p}$. (Ser108Leu)), was identified in MYT1, in a patient presenting with a severe form of OAVS. Functional studies showed that MYT1 overexpression downregulated all RA receptors genes (RARA, RARB, RARG), involved in RA-mediated transcription, whereas no effect was observed on CYP26A1 expression, the major enzyme involved in RA degradation, Moreover, MYT1 variants impacted significantly the expression of these genes, further supporting their pathogenicity. In conclusion, a third variant affecting function in MYT1 was identified as a cause of OAVS. Furthermore, we confirmed MYT1 connection to RA signaling pathway. European Journal of Human Genetics (2017) 25, 1083-1086; doi:10.1038/ejhg.2017.101; published online 14 June 2017

\section{INTRODUCTION}

Oculo-auriculo-vertebral spectrum (OAVS) is a developmental disorder characterized by hemifacial microsomia associated with ear, eyes and vertebrae malformations, showing highly variable expressivity. Various other organs malformations are commonly retrieved. ${ }^{1}$ Goldenhar syndrome (GS) could be considered as the most severe form of OAVS phenotype. ${ }^{2}$

Its etiology remains unknown even if environmental factors, including embryonic exposure to retinoic acid (RA) ${ }^{3}$ and genetic factors were documented. ${ }^{4,5}$ Recently, our group identified MYT1 (NM_004535.2) encoding the myelin transcription factor 1 as the first gene implicated in OAVS. Indeed, we found two heterozygous variants affecting function in MYT1, one nonsense de novo c.25C > T (p. $\left(\right.$ Arg $\left.\left.9^{*}\right)\right)$ and one inherited missense c.314C $>$ T (p.(Ser105Leu)), in two unrelated patients among a cohort of 169 OAVS patients. ${ }^{2}$

The recruitment of 57 new Brazilian OAVS patients allowed us to identify a novel variant in MYT1. Moreover, functional studies showed that the repressor role of MYT1 on several genes involved in the RA pathway was altered by missense variants affecting function.

\section{MATERIALS AND METHODS}

Fifty-seven patients were recruited from the Medical Genetic Center of the Genetic and Morphology Department of the Universidade Federal de São Paulo, Brazil. An ethics committee (CPP: DC2012/76) approved this study. SNP-arrays (genome-wide human SNP array 6.0, CytoScan Array 750k and HD, Affymetrix, Santa Clara, CA, USA) were performed in all patients.

Exons of the MYT1 gene were amplified using a standard protocol. ${ }^{2}$ PCR fragments were sequenced by GS Junior technology (ICM, Paris, France). Variants were confirmed by Sanger sequencing (Genewiz, France) and then registered in LOVD database (www.LOVD.nl/MYT1 under following individual
ID 00095945 and $00095942,00095943 / 00095955$ for variants previously described $^{2}$ ).

The pCS2+-MYT1 (MYT1-WT) was obtained from GeneCust (Ellange, Luxemburg). The MYT1-p.Ser108Leu and MYT1-p.Ser105Leu constructs corresponded to c.323C $>\mathrm{T}$ (p.Ser108Leu) and c.314C $>\mathrm{T}$ (p.Ser105Leu) variants, respectively, were generated by site-directed mutagenesis. ${ }^{2}$ Cell culture, transient transfections and RT-qPCR were performed as previously described. ${ }^{2}$ Quantitative expression of RARA, RARB, RARG and CYP26A1 was determined using the $2^{-\Delta \Delta}$ Ct method with GUSB and RPLPLO as reference genes (primers available on request).

\section{RESULTS}

All recruited patients presented hemifacial microsomia, characterized by mandibular/malar or maxillar hypoplasia, and microtia (including $23 \%$ with anotia), which was associated with preauricular tags (40\%) and/or dysplastic ears (63\%). Eye abnormalities were observed in $74 \%$ of patients mainly due to abnormal orbital position (63\%). Vertebral defects were found in $89 \%$ of patients. Furthermore, cardiac and renal anomalies were present in 47 and $16 \%$ of patients, respectively (Table 1). No pathogenic CNV were identified among this cohort. MYT1 screening revealed a de novo missense variant c. $323 \mathrm{C}>\mathrm{T}$ (p.(Ser108Leu)) in a GS patient born from unrelated and healthy parents (Figure 1a). Both parents did not carry the missense variant identified in the index patient. In silico analysis predicted a pathogenic effect of the c.323C $>\mathrm{T}$ (p. (Ser108Leu)) variant, which is reported 2/120 646 in ExAC database but notably it was not found in latino population. The patient presented a right anotia with preauricular tags and cervical pits, right stenosis of the external auditory meatus and a left grade III microtia (Figure $1 \mathrm{~b})$ with right conductive $(65-75 \mathrm{~dB})$ and left mixed (50-70 dB) hearing loss. Moreover, right hemifacial microsomia with moderate soft tissue deficiency, a small mandible and

\footnotetext{
${ }^{1}$ Laboratoire Maladies Rares: Génétique et Métabolisme (MRGM), U 1211 INSERM, University Bordeaux, Bordeaux, France; ${ }^{2}$ Division of Genetics, Department of Morphology and Genetics, Universidade Federal de Sao Paulo, Sao Paulo, Brazil; ${ }^{3} \mathrm{CHU}$ de Bordeaux, Service de Génétique Médicale, Centre de Référence Anomalies du Développement et Syndromes Malformatifs, Bordeaux, France

${ }^{*}$ Correspondence: Dr C Rooryck, Laboratoire Maladies Rares: Génétique et Métabolisme, CHU Pellegrin - Ecole des Sages-femmes, Place Amélie Raba-Léon, 33076 Bordeaux France. Tel: +33 5578203 57; Fax: +33 5567956 48; E-mail: caroline.rooryck-thambo@chu-bordeaux.fr Received 10 November 2016; revised 26 April 2017; accepted 2 May 2017; published online 14 June 2017
} 
Table 1 Clinical description of the 57 Brazilian patients compared to the cohort already published ${ }^{2}$ as reference and clinical pattern for the 3 OAVS patients presenting variants in MYT1

\begin{tabular}{|c|c|c|c|c|c|c|c|}
\hline \multirow{2}{*}{$\frac{\text { Description }}{\text { Total patients }}$} & \multicolumn{2}{|c|}{ Brazilian patients } & \multicolumn{2}{|c|}{ Described cohort ${ }^{2}$} & \multirow[t]{2}{*}{$\begin{array}{l}\text { Patient c. } 25 C>T \text {, } \\
\left(\text { p. }\left(\operatorname{Arg} 9^{*}\right)\right)^{2}\end{array}$} & \multirow[t]{2}{*}{$\begin{array}{c}\text { Patient c.314C >T, } \\
(\text { p. }(\text { Ser105Leu }))^{2}\end{array}$} & \multirow[t]{2}{*}{$\begin{array}{c}\text { Brazilian patient c. } 323 C>T \\
(p .(\text { Ser108Leu)) }\end{array}$} \\
\hline & 57 & $100 \%$ & 169 & $100 \%$ & & & \\
\hline Sex, F & 21 & $37 \%$ & 91 & $54 \%$ & + & - & + \\
\hline Sex, M & 36 & $63 \%$ & 78 & $46 \%$ & - & + & - \\
\hline OAVS familial history & 5 & $9 \%$ & 19 & $11 \%$ & - & - & - \\
\hline Twinning & 5 & $9 \%$ & 8 & $5 \%$ & - & - & - \\
\hline Gestational diabetes & 4 & $7 \%$ & 5 & $3 \%$ & - & - & - \\
\hline Psychomotor delay & 11 & $19 \%$ & 16 & $9 \%$ & - & - & - \\
\hline Ear abnormality & 57 & $100 \%$ & 166 & $98 \%$ & + & + & + \\
\hline Anotia & 13 & $23 \%$ & 18 & $11 \%$ & - & - & $+\mathrm{R}$ \\
\hline Microtia & 57 & $100 \%$ & 97 & $57 \%$ & $+\mathrm{R}$ & - & $+\mathrm{L}$ \\
\hline Preauricular Tag & 23 & $40 \%$ & 101 & $60 \%$ & $+\mathrm{R}$ & $+\mathrm{L}$ & $+\mathrm{L}$ \\
\hline Preauricular pit & 1 & $2 \%$ & 10 & $6 \%$ & - & - & $+\mathrm{L}$ \\
\hline Ear dysplasia & 36 & $63 \%$ & 126 & $75 \%$ & $+\mathrm{R}$ & $+\mathrm{L}$ & $+\mathrm{B}$ \\
\hline Hearing loss & 45 & $79 \%$ & 86 & $51 \%$ & + & + & + \\
\hline Conductive hearing loss & 40 & $70 \%$ & 47 & & $+\mathrm{R}$ & $+\mathrm{B}$ & $+\mathrm{R}$ \\
\hline Sensorineural hearing loss & 26 & $46 \%$ & 9 & $5 \%$ & - & - & - \\
\hline 'Mixed' Hearing loss & 21 & $37 \%$ & 6 & $4 \%$ & - & - & $+\mathrm{L}$ \\
\hline Hemifacial microsomia & 56 & $98 \%$ & 139 & $82 \%$ & & & \\
\hline Microsomia R & 31 & $54 \%$ & 38 & $22 \%$ & + & - & + \\
\hline Microsomia L & 23 & $40 \%$ & 41 & $24 \%$ & - & + & - \\
\hline Mandibular, malar, maxillar hypoplasia & 56 & $98 \%$ & 106 & $63 \%$ & + & + & + \\
\hline Eye abnormality & 42 & $74 \%$ & 64 & $38 \%$ & + & - & + \\
\hline Epibulbar dermoid & 7 & $12 \%$ & 42 & $25 \%$ & $+\mathrm{R}$ & - & - \\
\hline Coloboma & 5 & $9 \%$ & 16 & $9 \%$ & - & - & - \\
\hline Microphtalmia & 18 & $32 \%$ & 19 & $11 \%$ & - & - & - \\
\hline Abnormal orbital position & 35 & $61 \%$ & ND & ND & - & - & + \\
\hline Micrognathism/retrognathism & 44 & $77 \%$ & 31 & $18 \%$ & - & - & - \\
\hline Hypertelorism & 6 & $11 \%$ & 14 & $8 \%$ & - & - & - \\
\hline Other dysmorphia & 5 & $9 \%$ & 55 & $33 \%$ & - & - & - \\
\hline Macrocephaly, microcephaly & 2 & $4 \%$ & 0 & $0 \%$ & - & - & - \\
\hline Vertebral abnormality & 51 & $89 \%$ & 67 & $40 \%$ & + & + & + \\
\hline Cardiac malformation & 27 & $47 \%$ & 48 & $28 \%$ & $+(\mathrm{VSD})$ & - & $+(\mathrm{ASD} / \mathrm{VSD})$ \\
\hline Renal malformation & 9 & $16 \%$ & 14 & $8 \%$ & & & \\
\hline Cerebral malformation & 12 & $21 \%$ & 8 & $5 \%$ & + (seizures) & - & $\begin{array}{l}+ \text { (accentuation of } \\
\text { groove and cracks) }\end{array}$ \\
\hline Other abnormality & 34 & $60 \%$ & 16 & $9 \%$ & - & - & - \\
\hline Macrostomia & 27 & $47 \%$ & 19 & $11 \%$ & - & - & - \\
\hline Orofacial cleft & 18 & $32 \%$ & 42 & $25 \%$ & - & - & - \\
\hline
\end{tabular}

Abbreviations: + , presence of criterion; - , absence of criterion; ND, not determined.

a right glenoid fossa with short ramus mandibular branch involvement were observed (Figures $1 \mathrm{~b}$ and $\mathrm{c}$ ). She had an abnormal orbital position. Spinal X-rays revealed cervical vertebrae fusion with reduced spaces from C5 to C7, and from L3 to S1. She had atrial and ventricular septal defects. She also presented an accentuation of grooves and cracks of the brain, a sign of neonatal hypoxia, and a micrognathism (Table 1).

Following MYT1-WT overexpression, expressions of RARA, RARB and $R A R G$ were found decreased by $2.91-, 1.3$ - and a 3.84 -fold, respectively (Figures $2 \mathrm{a}-\mathrm{c}$ ). As a functional test, the effect of MYT1-p. Ser108Leu and MYT1-p.Ser105Leu (previously described ${ }^{2}$ ) overexpression on RARs expression was studied. Both mutants had a significantly lower inhibitory effect on RARA and RARG expression than MYT1-WT (Figures 2a and c). However, MYT1-p.Ser105Leu inhibited RARB expression to a significantly lesser extent than MYT1WT, while MYT1-p.Ser108Leu induced a significantly higher decrease (Figure 2b).

Additionally, we looked for the effect of MYT1 on a gene involved in RA-catabolism. MYT1-WT overexpression did not induce any significant change on CYP26A1 expression. However, MYT1-p.Ser105Leu increased its expression whereas MYT1-p.Ser108Leu form decreased it (Figure 2d).

\section{DISCUSSION}

We described here 57 new patients presenting with a typically heterogeneous OAVS. However, they presented a higher percentage of ocular and vertebral defects, and consequently, more patients were considered with GS than in previous reports: $40 \%$ versus $16 \%^{2}$ or $7.5 \% .^{6}$ 
a

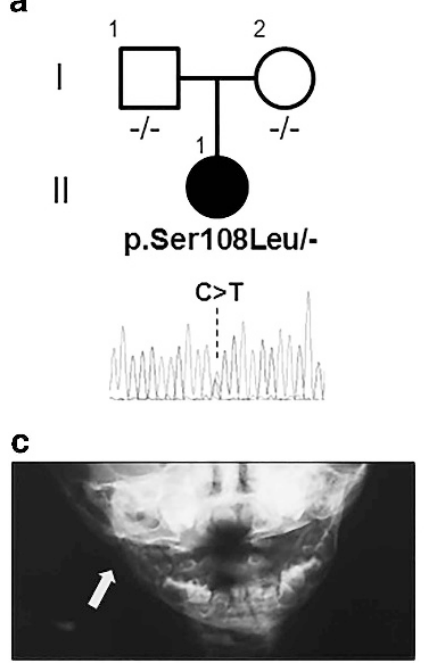

b
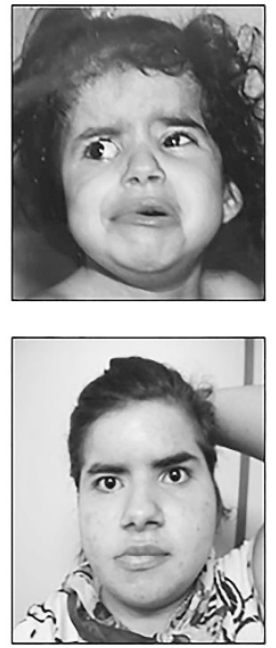
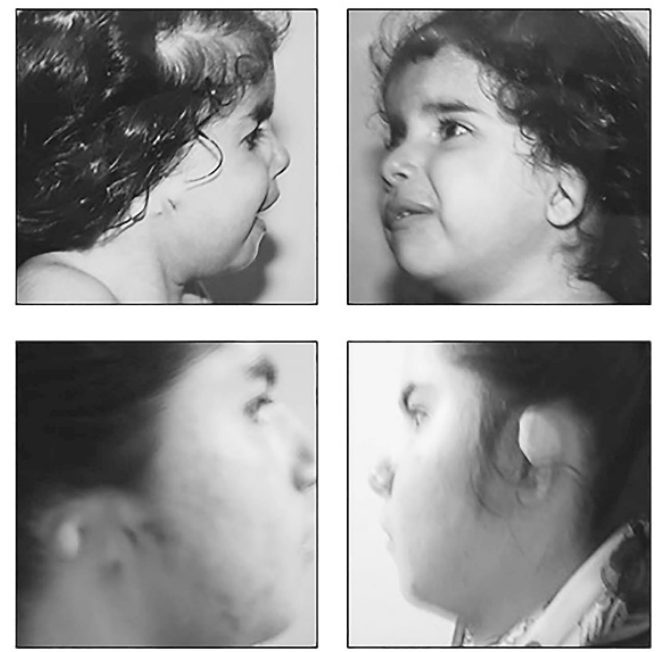

Figure 1 Pedigree and photographs of the patient carrying the c.323C >T (p.Ser108Leu) variant in MYT1. (a) Family tree and electrophoregram showing the de novo heterozygous missense variant. (b) Photographs of the proband showing facial asymmetry, right anotia and left microtia at age 1 and 17 years (top and bottom panels, respectively). (c) X-rays of proband at 1 year showing hypoplasia the right mandibular branch (arrow).
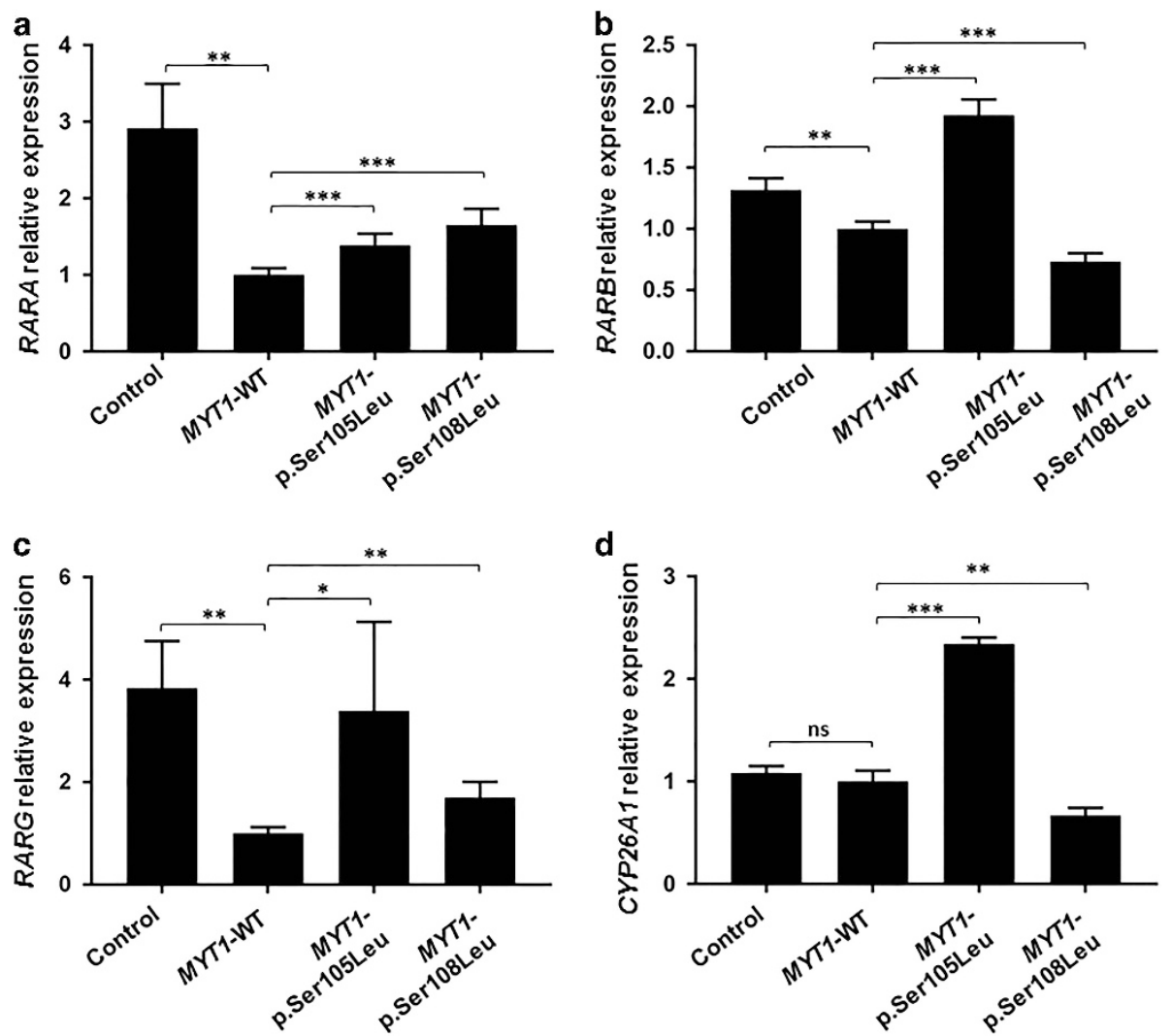

Figure 2 MYT1 variants altered regulation of RARs genes and CYP26A1. RT-qPCR analysis of RARA (a), RARB (b), RARG (c) and CYP26A1 (d) are shown following overexpression of either empty, MYT1-WT, MYT1-p.Ser105Leu or MYT1-p.Ser108Leu pCS2+ expression vector. The relative transcript level was calculated as fold change using the $2^{-\Delta \Delta C t}$ method $(n=3-6)$. Statistical significance was evaluated using $t$-test $\left({ }^{*} P<0.05 ;{ }^{*} P<0.01 ;{ }^{* *} P<0.001\right)$.

Recently, our group described MYT1, a transcription factor involved in neurogenesis, ${ }^{7}$ as the first gene involved in OAVS. ${ }^{2}$ In the present study, we identified a novel de novo missense variant affecting function, c.323C $>\mathrm{T}$ (p. (Ser108Leu)) in MYT1 in a GS patient. Interestingly, this new variant represents the same amino acid substitution (Serine into Leucine), only 3 residues next to the c. $314 \mathrm{C}>\mathrm{T}$ (p.(Ser105Leu)) variant. $^{2}$ Even if no functional domain was described, the close vicinity of these two mutated residues in OAVS patients suggests a potential role for this region.

Considering that embryonic RA exposure could induce OAVS features, we previously described that RA, the natural RAR ligand, induced MYT1 expression but also that MYT1 was a repressor of 
$R A R B$ expression in vitro. ${ }^{2}$ Very recently, it was shown that Myt1 bound genomic proximal region of Rara and repressed its expression, supporting a direct regulation. ${ }^{7}$ Thus, we further explored MYT1 effect on all RARs, and found that MYT1 had a repressor effect on their expression.

We thereby studied the effect of the two MYT1 missense variant affecting function on the expression of RARs. The overexpression of both mutated forms inhibited RARA and RARG expression to a lesser extent than MYT1-WT. However, the c.323C > T (p.Ser108Leu) variant inhibited RARB expression to a significantly higher extent than MYT1WT, whereas c.314C $>\mathrm{T}$ (p.Ser105Leu) had the opposite effect. ${ }^{2}$ Although these results appeared confusing, RARs dysregulations are involved into craniofacial defects either due to up- or downregulation. Indeed, loss and gain-of-function variants occurring in the $R A R B$ gene both cause anophthalmia and diaphragmatic hernia. ${ }^{8}$ Moreover, RARA expression was upregulated in fibroblasts from the secondary palate of a patient presenting a cleft/lip palate. ${ }^{9}$ Additionally, treatment with specific Rara or Rarb agonists led to craniofacial abnormalities in murine embryos ${ }^{10,11}$ and injection of Rarg dominant negative mRNA in Xenopus embryos led to a partial resistance to RA. ${ }^{12}$

Subsequently, we investigated the effect of MYT1 on genes involved in RA degradation, thus regulating RA availability. In HEK293 (human embryonic kidney) cells, CYP26A1 is the major enzyme of RA catabolism. ${ }^{13}$ MYT1-WT overexpression had no detectable impact on CYP26A1 expression. As observed for RARB expression, MYT1-p. Ser105Leu overexpression led to an upregulation of CYP26A1 expression, whereas MYT1-pSer108Leu led to a downregulation of both genes. Therefore, the deregulation of RARB by MYT1-mutated forms seems to be compensated by CYP26A1 deregulation in order to control RA availability, thus restoring a correct RA signaling pathway. Rarb and Cyp26a1 were shown to be upregulated after RA treatment in murine embryo, ${ }^{14}$ thus supporting a regulating loop between these two genes. Moreover, downregulation of Rarb and Cyp26a1 (but also of Rara $)^{15}$ was observed in LgDel-22q11 mice and patients with OAVS features carried $22 \mathrm{q} 11$ deletions. ${ }^{5,16}$ As shown with RARG, ${ }^{17}$ our results suggest that RARB could participate in CYP26A1 regulation. Moreover, Cyp26A1 1 - embryos present malformations of the hindbrain, ${ }^{18}$ where Myt1, Cyp26A1 and Rarb are expressed, ${ }^{18,19}$ and where RA signaling pathway activity is detected. ${ }^{20}$

Taking together, our results show that MYT1 regulates genes involved in the RA pathway, and thus participates in its negative feedback. Variants affecting function in MYT1 could lead to a disruption of RA signaling and may be responsible for OAVS features. Partial contradictory effects of the two MYT1 variants could be associated by the paradoxical teratogenic mechanism evoked for RA. Indeed, ATRA exposure leads to rapid changes in enzymes involved in RA metabolism and induces a longer-term RA-deficiency. ${ }^{14}$ Thus, future investigations will allow to better understand how variants in MYT1 impact RA signaling, and especially if OAVS features could be related to one specific RAR or to the combined effect of them but also to study how MYT1 could modulate the expression of genes involved in RA-synthesis due to the importance of Raldhs in embryonic development. ${ }^{21,22}$

In conclusion, we reported a novel de novo variant in MYT1 gene, thus confirming its involvement in OAVS. The low frequency of variants ( 1 out of 57 and 2 out of $169^{2}$ ) supports the high genetic heterogeneity of this spectrum. The identification of new candidate genes will increase the understanding of the syndrome and more broadly of embryonic development processes. In particular, genes involved in RA pathway could be a good target for future investigation in OAVS. ${ }^{22}$

\section{CONFLICT OF INTEREST}

The authors declare no conflict of interest.

\section{ACKNOWLEDGEMENTS}

This work was supported by the ANR (Agence Nationale pour la Recherche, ANR-12-JVS1-0002), the University Hospital of Bordeaux (Appel Offre Interne GOLDGEN 2012), the Fondation Maladies Rares, and the Ministry of Research and Higher Education (PhD fellowship for $\mathrm{MB}$ ) and São Paulo Research Foundation (FAPESP), Brazil. We thank Mr MARIE Yannick (in ICM in La Pitié Salpêtrière Hospital, Paris, France) for his technical support during sequencing by GS Junior Technology. We also thank the patients and their families.

1 Barisic I, Odak L, Loane M et al: Prevalence, prenatal diagnosis and clinical features of oculo-auriculo-vertebral spectrum: a registry-based study in Europe. Eur J Hum Genet 2014; 22: 1026-1033

2 Lopez E, Berenguer M, Tingaud-Sequeira A et al: Mutations in MYT1, encoding the myelin transcription factor 1 , are a rare cause of OAVS. J Med Genet 2016; 53: $752-760$.

3 Lammer EJ, Chen DT, Hoar RM et al: Retinoic acid embryopathy. N Engl J Med 1985; 313: 837-841.

4 Rooryck C, Souakri N, Cailley D et al: Array-CGH analysis of a cohort of 86 patients with oculoauriculovertebral spectrum. Am J Med Genet 2010; 152A: 1984-1989.

5 Beleza-Meireles A, Clayton-Smith J, Saraiva JM, Tassabehji M: Oculo-auriculo-vertebral spectrum: a review of the literature and genetic update. J Med Genet 2014; 51: 635-645.

6 Tasse C, Böhringer S, Fischer S et al: Oculo-auriculo-vertebral spectrum (OAVS): clinical evaluation and severity scoring of 53 patients and proposal for a new classification. Eur J Med Genet 2005; 48: 397-411.

7 Vasconcelos FF, Sessa A, Laranjeira C et al: MyT1 counteracts the neural progenitor program to promote vertebrate neurogenesis. Cell Rep 2016; 17: 469-483.

8 Srour M, Chitayat D, Caron V et al: Recessive and dominant mutations in retinoic acid receptor beta in cases with microphthalmia and diaphragmatic Hernia. Am J Hum Genet 2013; 93: 765-772.

9 Baroni T, Bellucci C, Lilli $\mathrm{C}$ et al: Retinoic acid, GABA-ergic, and TGF-beta signaling systems are involved in human cleft palate fibroblast phenotype. Mol Med Camb Mass 2006; 12: 237-245.

10 Elmazar MMA, RühI R, Reichert U, Shroot B, Nau H: RAR $\alpha$-mediated teratogenicity in mice is potentiated by an rxr agonist and reduced by an RAR antagonist: dissection of retinoid receptor-induced pathways. Toxicol Appl Pharmacol 1997; 146: 21-28.

11 Matt N, Ghyselinck NB, Wendling O, Chambon P, Mark M: Retinoic acid-induced developmental defects are mediated by RAR $\beta / R X R$ heterodimers in the pharyngeal endoderm. Development 2003; 130: 2083-2093.

12 Smith DP, Mason CS, Jones E, Old R: Expression of a dominant negative retinoic acid receptor $\gamma$ in Xenopus embryos leads to partial resistance to retinoic acid. Rouxs Arch Dev Biol 1993; 203: 254-265.

13 Ross AC, Zolfaghari R: Cytochrome P450s in the regulation of cellular retinoic acid metabolism. Annu Rev Nutr 2011; 31: 65-87.

14 Lee LMY, Leung C-Y, Tang WWC et al: A paradoxical teratogenic mechanism for retinoic acid. Proc Natl Acad Sci 2012; 109: 13668-13673.

15 Maynard TM, Gopalakrishna D, Meechan DW, Paronett EM, Newbern JM, LaMantia A-S: $22 q 11$ Gene dosage establishes an adaptive range for sonic hedgehog and retinoic acid signaling during early development. Hum Mol Genet 2013; 22: 300-312.

16 Digilio MC, McDonald-McGinn DM, Heike $\mathrm{C}$ et al: Three patients with oculo-auriculovertebral spectrum and microdeletion 22q11.2. Am J Med Genet A 2009; 149A: 2860-2864.

17 Colovati MES, Bragagnolo S, Guilherme RS et al: Atypical 581-kb 22q11.21 deletion in a patient with oculo-auriculo-vertebral spectrum phenotype. Cytogenet Genome Res 2015; 147: 130-134.

18 Loudig O, Babichuk C, White J, Abu-Abed S, Mueller C, Petkovich M: Cytochrome P450RAI(CYP26) promoter: a distinct composite retinoic acid response element underlies the complex regulation of retinoic acid metabolism. Mol Endocrinol 2000; 14: 1483-1497.

19 Abu-Abed S, Dollé P, Metzger D, Beckett B, Chambon P, Petkovich M: The retinoic acid-metabolizing enzyme, CYP26A1, is essential for normal hindbrain patterning, vertebral identity, and development of posterior structures. Genes Dev 2001; 15: 226-240.

20 Matsushita F, Kameyama T, Kadokawa Y, Marunouchi T: Spatiotemporal expression pattern of Myt/NZF family zinc finger transcription factors during mouse nervous system development. Dev Dyn Off Publ Am Assoc Anat 2014; 243: 588-600.

21 Dollé P, Fraulob V, Gallego-Llamas J, Vermot J, Niederreither K: Fate of retinoic acidactivated embryonic cell lineages. Dev Dyn 2010; 239: 3260-3274.

22 Duester G: Retinoic acid regulation of the somitogenesis clock. Birth Defects Res Part C Embryo Today Rev 2007; 81: 84-92. 